\title{
Obesidad y Adolescencia: Exploración de Aspectos Relacionales y Emocionales
}

\section{Obesity and Adolescence: Exploration of Relational and Emotional Aspects}

\author{
Daniela Carrasco Dell'Aquila* \\ Elena Gómez Castro \\ Antonia Staforelli Mosca \\ Universidad Diego Portales, Santiago, Chile. \\ (Rec: 11 de junio de 2008 Acep: 15 abril 2009)
}

\begin{abstract}
Resumen
El propósito de la investigación fue la exploración de factores emocionales en adolescentes que presentan obesidad y a raíz de los hallazgos encontrados hacer recomendaciones psicoterapéuticas. Para conocer la vida emocional y las relaciones significativas de estos adolescentes se aplicaron cuatro instrumentos diagnósticos: Entrevista clínica, TRO, PBI y OQ45, a dos grupos de adolescentes con y sin diagnóstico de obesidad, grupo de estudio y grupo de control respectivamente, con un $n=25$. Los resultados de esta investigación muestran que los adolescentes obesos presentan tanto sintomatología psíquica, ansiedad y depresión, como una dificultad en la tolerancia y manejo de afectos intensos relacionada con fallas anteriores provenientes del contexto parental.
\end{abstract}

Palabras claves: Obesidad, adolescencia, psicosomática.

\begin{abstract}
The purpose of the research was to explore emotional factors in adolescents with obesity and according to the findings to make psychotherapeutic recommendations.

To know the emotional life and significant relationships of these adolescents four diagnostic instruments were applied: a clinical interview, TRO, PBI and OQ45, on two groups of adolescents (with and without obesity diagnosis, study group and control group respectively), with an $n=25$.

The results showed that obese adolescents presented psychological symptoms, anxiety and depression, as well as a difficulty tolerating and handling intense emotions related to previous failures coming from the parental context.
\end{abstract}

Key words: Obesity, adolescence, psychosomatic 


\section{Introducción}

El presente trabajo corresponde a los resultados obtenidos en un proyecto de investigación financiado durante el año 2006 por la Universidad Diego Portales. El propósito de la investigación fue la exploración de factores emocionales en adolescentes que presentan obesidad y a raíz de los hallazgos encontrados hacer recomendaciones psicoterapéuticas.

La obesidad en Chile se ha transformado en los últimos 10 años en un problema de salud pública, que afecta no sólo a la población adulta sino también a los niños pequeños y adolescentes. Esta condición patológica se encuentra asociada a una serie de factores, destacándose aquellos socioculturales como son los hábitos alimenticios, la vida sedentaria y la falta de espacio físico para actividades recreativas. Entre los factores genéticos se plantea controversialmente que madres obesas tienden a tener hijos obesos. Respecto de los factores emocionales, la mayoría de los estudios en Chile y en el extranjero señalan la presencia e influencia de éstos, pero no se especifica mayormente al respecto.

Las cifras de prevalencia de la obesidad en adolescentes en Chile señalan que en los últimos 12 años se ha producido un aumento del 1,5\% por año, lo que da la alarmante situación de que uno de cada 4 niños es obeso y uno de cada 3 corre el riego de serlo (INTA). Se plantea que la prevalencia de la obesidad en el segmento 6-16 años ha aumentado de un $5 \%$ a un $25 \%$ en hombres y de un $10 \%$ a un $27 \%$ en mujeres. Solo países como USA y Japón tiene, cifras similares a las nuestras, con la preocupante diferencia que han alcanzado estas cifras en períodos de años mayores que las nuestras (USA en 30 años, Japón en 20 años y Chile en 10 años). Los estudios internacionales señalan que un niño obeso tiene un $40 \%$ de posibilidades de ser un adulto obeso con las negativas consecuencias para su salud futura.

Para esta investigación se consideró a la adolescencia como una etapa del desarrollo humano que presenta una serie de desafíos en la indispensable adaptación que el adolescente debe hacer frente a sí mismo, frente a su cuerpo $\mathrm{y}$ frente al medio. A su vez, la adolescencia fue considerada una etapa en la que resulta difícil diagnosticar salud y normalidad, ya que la propia adolescencia modifica la forma en que se presentan lo que denominamos trastornos o enfermedades.

La presencia de obesidad en la adolescencia (descartando la presencia primaria de otras patologías somáticas) fue conceptualizada desde una perspectiva en la que el cuerpo es pensado como una unidad psicosomática y nos planteamos la interrogante si la obesidad podía ser considerada como la expresión de dificultades de autorregulación de estados afectivos inconscientes y preconscientes, frente a los cuales la alimentación surgiría como una forma de lograr un estado interno de equilibrio emocional. Nos interesaron, por lo tanto, en la historia personal de los adolescentes obesos, los aspectos de su vida emocional y de sus relaciones afectivas significativas que nos permitiesen comprender el cuerpo y la obesidad que lo enferma.

Es posible encontrar muchas definiciones acerca del concepto de psicosomática según de qué disciplina sea la que hable. Una de las que puede considerarse es aquella que plantea que "el objeto de la clínica psicosomática son las enfermedades físicas en cuyo determinismo o en cuya evolución influyen factores psíquicos o conflictivos" (Kreisler, Fain \& Soulé, 1974).

Esta es una definición amplia que sin embargo nos permite el intento de comprender una serie de manifestaciones corporales. En el psicoanálisis existen variadas formas de enfocar los fenómenos psicosomáticos y subrayaremos sólo algunas de ellas, para luego definir nuestro enfoque en esta investigación.

Cronológicamente se pueden distinguir a los autores de la Escuela de Chicago (Dumbar \& Alexander) como los pioneros en el desarrollo de este tema. Otro autor destacado es D. W. Winnicott. También han tenido gran interés los planteamientos de la Escuela Psicosomática de París (Marty, Kreisler \& Fain), los de una autora como J. Mc Dougall y actualmente el planteamiento hecho en psicoanálisis por las teorías relacionales e intersubjetivas (Mitchell, S. Stolorow, R. \& Atwood, G. 1993).

La escuela de Chicago fue la que realizó las primeras investigaciones sistemáticas en el campo de las enfermedades psicosomáticas $\mathrm{y}$, considerando las concepciones freudianas de neurosis propuso la noción de "neurosis de órgano" para referirse, a aquellos fenómenos en los que el sistema visceral neurovegetativo sería la sede de las enfermedades psicosomáticas. De este planteamiento surgieron las clásicas enfermedades psicosomáticas (7) que como tales fueron integradas a la medicina (asma bronquial, hipertensión arterial esencial, artritis reumatoídea, rectocolitis hemorrágica, úlcera duodenal, neurodermatosis, hipertiroidismo).

La Escuela psicosomática de París ha intentado elaborar una teoría unitaria de la organización psicosomática en la que subrayan la no especificidad de las situaciones conflictivas que se encuentran en el desencadenamiento de las afecciones psicosomáticas. Los autores de esta Escuela utilizan el término "pensamiento operatorio", el que podría considerarse como equivalente al concepto de alexitimia (utilizado por la Escuela de Chicago), para referirse a un tipo de pensamiento que se aferra a cosas y no a productos imaginativos ni a expresiones simbólicas. Este concepto sugiere la precariedad de la conexión con las palabras. Marty y Muzan (1992) sostienen el supuesto básico que los sucesos y situaciones que se presentan tocan nuestra afectividad y desencadenan una serie de excitaciones a las que debe darse una salida adecuada. Una buena parte de este trabajo corresponde a la elaboración mental siempre y cuando el individuo esté dotado de un buen soporte para la mentalización. El proceso de mentalización se construye 
gracias a que las representaciones cosas, a través del proceso simbólico, se van organizando progresivamente en representaciones palabras. Estas permiten el acceso a la fantasía, a la producción onírica o a los procesos de reflexión interna. Si se dan fallas o insuficiencias en la adquisición de representaciones palabras se corre el riesgo de que la vía somática aparezca de manera patológica.

Otro concepto utilizado por esta escuela es el de "depresión esencial”, el que fue esbozado por primera vez en 1963 como el de una depresión sin objeto. En esta depresión, el sentimiento de desvalorización personal y de herida narcisista se orienta hacia la esfera somática. Para estos autores, este cuadro pone en evidencia la precariedad del trabajo mental. En "La psicosomática del adulto", P. Marty (1992) plantea lo siguiente: "la depresión psicosomática, a la que en diversas ocasiones he llamado depresión sin objeto, en definitiva sería mejor llamarla depresión esencial, puesto que ella constituye la esencia misma de la depresión a saber, la disminución del nivel del tono libidinal sin contrapartida económica positiva alguna" (Marty, P. 1990).

A partir de estos planteamientos, esta Escuela plantea la existencia de una economía psicosomática tomando el preconsciente como un punto central. Así mientras más rico sea el preconsciente en representaciones relacionadas entre sí, más probabilidades tendrá una patología eventual de ubicarse en el plano mental. En cambio mientras menos rico sea en ellas, más riesgo existe que la patología se ubique en el plano somático.

Por otra parte, desde una visión basada en una vasta experiencia clínica con pacientes somáticos en psicoanálisis, J. MacDougall (1991) se refiere al tema planteando que los fenómenos del cuerpo enfermo cumplen un papel defensivo, remontándose esta defensa a las fases tempanas del desarrollo en la cual la distinción entre sujeto y objeto aún no está bien establecida.

Esta autora destaca también las interacciones entre la madre y el lactante dándole gran importancia a esta relación diádica y a la posible comunicación entre ellos. De este modo, si hay deficiencias en la comunicación entre el bebé y su madre, éstas se manifestarán, a menudo y desde los primeros meses, de un modo psicosomático, siendo ésta la vía de expresión de los conflictos psíquicos. Las primeras funciones fisiológicas afectadas serían: la respiración, la digestión, la evacuación y el sueño.

Las observaciones de Mc Dougall (1991) en sus pacientes, la hicieron comprender que, en ciertas ocasiones, sus pacientes adultos funcionaban como niños pequeños al no poder utilizar las palabras como vehículo del pensamiento. No podían reaccionar más que al modo psicosomático frente a una emoción dolorosa.

Para un psicoanalista como D. W. Winnicott (1954), cada individuo poseería una tendencia heredada a alcanzar la unidad psique-soma (guión que articula y separa a la vez), es decir, hacia una identidad experiencial de la psique y la totalidad del funcionamiento corporal. Esta tendencia lleva al bebé y al niño hacia un cuerpo en funcionamiento sobre el cual y desde el cual se desarrolla la personalidad completa. Cuando esta unidad psique-soma no se alcanza el individuo puede recurrir a una defensa, la escisión. La verdadera enfermedad psicosomática es la escisión entre psique y soma, es la pérdida de la integración que conduce a la separación entre el cuidado físico y el cuidado de la psique. Así entonces, la enfermedad psicosomática sería el negativo de un positivo, con ello este autor se está refiriendo a que en la enfermedad psicosomática se ha roto la tendencia hacia la integración.

En esta concepción de psique-soma, el logro de la "residencia" de la psique en el cuerpo procede de lo personal y de lo ambiental. Con el término "personalización", Winnicott (1949) se refiere al logro de la unidad psique-soma y éste en la infancia depende de la capacidad de la madre de sumar su participación emocional a la que originalmente es física y fisiológica. La conservación de la relación entre cuerpo y psique es definido como salud: "debemos asumir que la salud en el desarrollo temprano del individuo, se relaciona con la continuidad del existir. El temprano psique-soma procede de una cierta línea de desarrollo en la que su continuidad de existir no ha sido perturbado. El inicio de la existencia es psicosomático. En otras palabras, para el saludable desarrollo es necesario un ambiente perfecto. Al comienzo la necesidad es absoluta" (Winnicott D. 1949, pp. 245).

Por lo tanto, para este autor la enfermedad psicosomática es siempre el síntoma de que "algo" no anduvo bien en el desarrollo emocional temprano, sin embargo, siempre queda en pie la posibilidad de no perder para siempre el nexo psicosomático.

Consideramos que en esta concepción se refleja una noción que le da una gran importancia a los procesos que en un individuo dependen de la relación con otros significativos.

\section{La Comprensión de la Enfermedad Psicosomática desde una Perspectiva Relacional e Intersubjetiva.}

S. Mitchell, (1993) autor de la Teoría Relacional en psicoanálisis, plantea que los límites y relaciones entre el cuerpo y la mente toman forma en un contexto relacional específico. La visión del ser humano en el paradigma relacional es concebirlo como conformado por una matriz de relaciones, de ahí que el cuerpo y la relación de éste con la mente sólo se puede comprender desde esta matriz relacional.

R. Stolorow y G. Atwood (1992) plantean desde su perspectiva intersubjetiva, que los límites entre la experiencia de la mente y del cuerpo son producto del contexto intersubjetivo. Desde esta mirada la dialéctica entre mente y cuerpo no puede ser entendida de manera aislada, esta 
dialéctica es siempre una propiedad del "sistema de vida". Para estos autores es imprescindible considerar al cuerpo y la mente en experiencia.

Esto ocurre por ejemplo con los afectos, ya que en el ámbito de las experiencias afectivas los límites que separan la mente y el cuerpo están siempre originados en una situación intersubjetiva.

Desde esta misma perspectiva, H. Krystal (1988) plantea que se debe considerar que los afectos evolucionan, es así que las tempranas experiencias son sólo sensaciones corporales y éstas posteriormente alcanzan la forma de estados subjetivos, los que pueden ser articulados verbalmente. Si se observa con atención, este es un camino absolutamente inverso al pensamiento de las primeras explicaciones respecto de la relación mente cuerpo. En los planteamientos psicoanalíticos iniciales, la pregunta consistía en cómo los procesos mentales pasaban a expresarse en el cuerpo, y el giro que estamos haciendo implica considerar que los afectos son en primer lugar sensaciones corporales y posteriormente eventos mentales.

Para Cristal (1988), este recorrido es un proceso maduracional y este autor plantea que son de gran importancia algunas formas de relación entre el niño y las personas que le rodean. Una de ellas es la habilidad de los cuidadores en identificar y verbalizar correctamente los tempranos afectos del niño, ya que así se produce la gradual integración de las experiencias.

Desde este punto de vista, la forma en que una persona llega a experienciar los afectos en la mente y no solamente en el cuerpo depende entonces de la presencia de un contexto facilitador intersubjetivo.

Lo que ocurre en ausencia de este contexto facilitador es que los afectos continuarán siendo experienciados primariamente como estados corporales. A partir de este punto se pueden dar dos caminos. Uno de ellos es que estos afectos queden en lo que en la teoría intersubjetiva desarrollada por R. Stolorow y G. Atwood (1992) corresponde al inconsciente no validado, es decir, no adquieren el carácter de una experiencia y, por lo tanto, en ausencia de una respuesta validadora, la persona nunca será capaz de articular simbólicamente sus estados. Esta experiencia no formulada es lo que Krystal ha denominado alexitimia.

Esta misma perspectiva se refleja en la actualidad con las nociones derivadas de la teoría intersubjetiva en psicoanálisis. Para los representantes de estas teorías (Stolorow R., Atwood G, Orange D. 1992), los límites entre la experiencia de la mente y el cuerpo son productos del contexto intersubjetivo, así la dialéctica entre mente y cuerpo no puede ser entendida de manera aislada, ésta (dialéctica) es propiedad del "Sistema de vida". Desde este punto de vista la manera en que una persona llega a experienciar los afectos depende directamente de la presencia de un contexto facilitador intersubjetivo.
En el desarrollo del ser humano se parte inicialmente de los afectos, los que inicialmente son sólo sensaciones corporales que luego evolucionan y se transforman en estados subjetivos, los que luego serán articulados verbalmente. Éste sería un recorrido en el que hay una estrecha dependencia de la forma en que las personas que rodean y cuidan al niño le facilitan la habilidad para identificar y verbalizar correctamente los tempranos afectos del niño y el logro de la integración de las experiencias. La función del cuidador primario es la de integrar firmemente las experiencias afectivas del niño y permitir que éste desarrolle la tolerancia a los afectos y el uso de ellos como señales para el self (sí mismo).

Lo que ocurre en la ausencia de un contexto facilitador intersubjetivo es que los afectos continúan siendo experienciados primariamente como estados corporales. Si la mayoría de las experiencias tempranas afectivas son sobre todo experiencias físicas es entonces la capacidad del cuidador (del niño) de sintonizar con los estados del niño lo que permitirá que éste reconozca sus estados. El déficit temprano en esta sintonización producirá una variedad de deformaciones. Una de ellas puede ser la falla en la residencia psicosomática (concepto estrechamente ligado a lo planteado por D. W. Winnicott), son estas últimas nociones: psique-soma, residencia psicosomática, contexto intersubjetivo facilitador, las que consideramos como concretas guías en nuestra investigación.

\section{Método}

Para conocer la vida emocional y las relaciones significativas de estos adolescentes se utilizaron instrumentos tales como la entrevista clínica, el T.R.O (Test de Relaciones Objetales) y el PBI (Test de Apego o Parental Bonding Instrument). Se buscó también detectar la presencia de sintomatología psíquica, a través de la aplicación del O.Q45 (el que detecta la presencia de síntomas angustiosos y depresivos). Todos estos instrumentos se encuentran validados en Chile.

A pesar que en las ciencias humanas para realizar una investigación (cualitativa) bastaría con los hallazgos encontrados en uno o en pocos casos, nos pareció interesante el contraste que podía existir entre una población de adolescentes obesos y los que no lo son. Para ello aplicamos los mismos instrumentos a un grupo de adolescentes que no presentaban obesidad. Es así que los hallazgos de la investigación están basados en dos grupos de adolescentes, cada uno de ellos conformado por 25 jóvenes entre 14 y 18 años, todos de estrato socioeconómico medio, siendo los adolescentes obesos consultantes de un Centro Hospitalario de la Región Metropolitana. 


\section{Resultados}

1.- Historia del Trastorno Corporal en el grupo de 25 adolescentes obesos.

- El $60 \%$ de la muestra presenta trastorno de peso hace más de 2 años. El 20\% lo ha tenido siempre y el 20\% sólo recientemente, es decir, hace menos de 2 años.

Figura 1:

Aumento de Peso Adolescentes Obesos

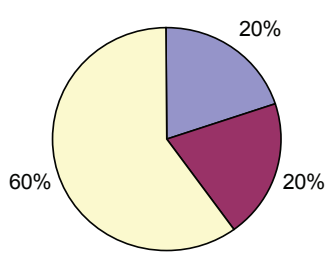

$\square$ Siempre

$\square$ Recientemente (2 años)

$\square$ Más de 2 años

- $\mathrm{Al} \mathrm{100 \%} \mathrm{le} \mathrm{preocupa} \mathrm{el} \mathrm{sobrepeso.}$

Figura 2:

Actitud ante la Obesidad

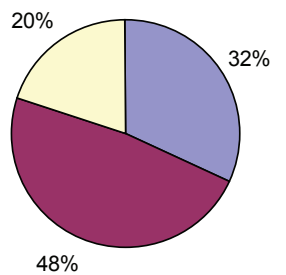

- El $48 \%$ tiene una actitud pasiva frente a la obesidad, el $20 \%$ es de negación y sólo el $32 \%$ tiene una actitud activa.

- El 64\% relaciona el sobrepeso con algún acontecimiento importante de su historia de vida. Estos acontecimientos importantes son:

$>$ Duelos por muerte en la familia cercana (abuelos, padre, madre).

$>$ Accidentes en la familia cercana.

$>$ Enfermedades en la familia cercana.

$>$ Cambios en el sistema de vida (cambio de ciudad, de colegio).

Los adolescentes vinculan estos acontecimientos de pérdidas afectivas a sentimientos depresivos.

2.- Respecto de la presencia de sintomatología psíquica en el grupo de adolescentes con obesidad (25). Resultados obtenidos con el Test OQ45.

Se encontraron diferencias en ambos grupos, es así que el grupo de adolescentes obesos presentó mayores índices de ansiedad y depresión y mayores dificultades en sus relaciones cercanas tanto familiares como sociales.

Figura 3.

Puntajes Altos OQ - 45.2

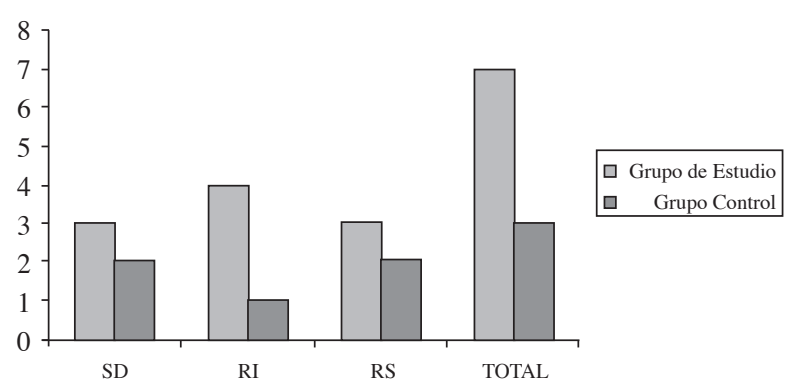

En términos comparativos, en relación al grupo control, se puede establecer que el grupo de adolescentes obesos presenta un elevado nivel de disconfort en su calidad de vida en general, tanto en lo referente a síntomas, dificultades en sus relaciones interpersonales y en su rol social.

Esto significa que comparativamente el grupo de estudio presenta un puntaje más alto en cuanto a presencia de sintomatología ansiosa y depresiva, como también desórdenes de ajustes y stress.

En las relaciones interpersonales, el grupo de adolescentes obesos presenta un puntaje mínimamente mayor que el grupo control. Esto significa que presentarían algunas dificultades en sus relaciones más cercanas donde habría quejas de soledad, conflictos con otros y problemas con la familia.

En cuanto al rol social, el grupo de adolescentes obesos presenta un puntaje también mínimamente mayor que el grupo control. Lo que indicaría dificultades en el ajuste del rol social, en este caso de estudiante, lo que daría stress e ineficacia en este rol.

3.- Respecto de la presencia de factores emocionales y patrones relacionales (apego) asociados al desarrollo del trastorno, en el grupo de adolescentes con obesidad (25). Resultados obtenidos con la aplicación del test PBI.

Encontramos un predominio de un vínculo de control sin afecto, el cual refiere a padres emocionalmente fríos, indiferentes y negligentes, además de ser controladores, intrusivos, con un contacto excesivo que infantiliza y dificulta la conducta autónoma de sus hijos.

- El 28\% de las madres y el 32\% de los padres del grupo de estudio presentan este tipo de vínculo. En cambio, en el grupo control sólo el $12 \%$ de las madres presenta este tipo de vínculo y un $4 \%$ en los padres.

- En el grupo de estudio, hay una percepción de cuidados bajos en un $40 \%$ en relación a las madres y en un $50 \%$ en relación a los padres. 
- La sobreprotección es percibida como alta en un 52\% para la madre y en un $40 \%$ para los padres.

Figura 4:

Percepción Sobreprotección Materna

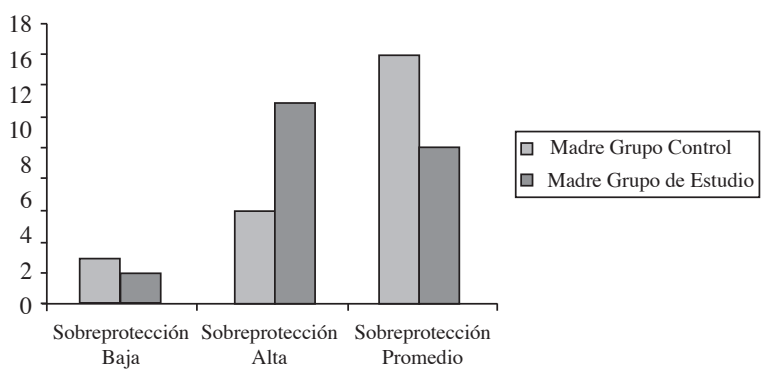

Figura 5:

Percepción Sobreprotección Paterna

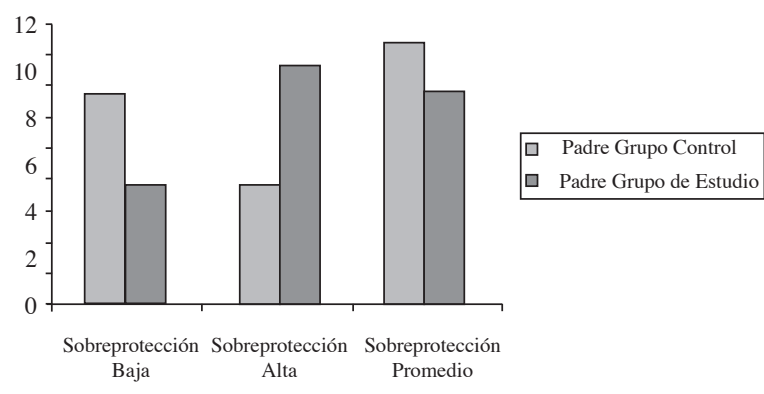

Si se comparan estos datos con el grupo control, la diferencia es significativa ya que solo el $24 \%$ percibe con alta sobreprotección a la madre y el $20 \%$ al padre.

- En el grupo de estudio, el 60\% define la relación con el padre como insatisfactoria, en tanto en el grupo control lo hace el $44 \%$.

- El 56\% define la relación con la madre como insatisfactoria, y en el grupo control solo el $24 \%$ la considera de esa forma.

- El 56\% de los adolescentes con obesidad opina que está insatisfecho con su familia y el 36\% del grupo control dice lo mismo.

4.- Respecto de la configuración de las relaciones afectivas significativas en el grupo de adolescentes con obesidad (25).

Resultados obtenidos con la aplicación del test de relaciones objetales (TRO):

- Aparecen elementos depresivos.

- La presencia de angustia es alta en ambos grupos. Pero en el grupo de los con sobrepeso destaca la presencia de mecanismos de defensa bajos (el 64\% de ello presenta este tipo de mecanismos en comparación con sólo un $20 \%$ en el grupo control).
- La dificultad para tolerar la exclusión se presenta en un $84 \%$ en el grupo con sobrepeso y en un $56 \%$ en el grupo control.

- Hay dificultad para integrar lo agresivo. Sólo el 2\% logra integrarlo, contra un 36\% que sí lo logra en el grupo control.

- El 24\% de los adolescentes con sobrepeso ha consultado a psicólogo y dentro de este $24 \%$, el $67 \%$ lo hace por síntomas depresivos.

\section{Discusión}

Una vez analizados los resultados podemos plantear que en los adolescentes obesos nos encontramos con un grupo de jóvenes que presentan una historia de aumento de peso de larga data, y que presentan en la actualidad un alto disconfort en su vida asociado a la presencia de reacciones de ansiedad y a manifestaciones depresivas. Estos hallazgos se encuentran asociados a factores provenientes del contexto intersubjetivo que los rodea, específicamente a un contexto parental caracterizado por una distancia emocional encubierta por la presencia de un excesivo control en las actividades y conductas del adolescente.

Los postulados teóricos de este proyecto plantean que la relación con el cuerpo (de unión o de escisión) está en estrecha dependencia del contexto relacional e intersubjetivo, es decir, de la relación que se establece entre el niño, púber o adolescente con el sistema de cuidado que lo rodea. En lo esencial, esto quiere decir que tanto la confirmación de la experiencia corporal, como la sintonización afectiva forman parte de un proceso que resulta ser primordial para el reconocimiento y luego la expresión de los afectos. Destacamos de estos planteamientos teóricos el énfasis sobre las tempranas experiencias del niño, las cuales en sus inicios serían sólo sensaciones corporales y posteriormente alcanzarían la forma de estados subjetivos, los que pueden ser articulados verbalmente. Desde este punto de vista, para que una persona llegue a experienciar los afectos en la mente y no solamente en el cuerpo se debe dar un proceso en el que un contexto facilitador intersubjetivo permita que las emociones y los afectos no sean disruptivos, sino que por el contrario puedan ser integrados a la unidad psique-soma.

Lo que ocurre en ausencia de dicho contexto es que los afectos continúan siendo experienciados primariamente como estados corporales, es decir, el cuerpo separado del psique es el único lugar a través del cual se manifiestan emociones y afectos.

Todo lo anterior nos condujo a observar con detención, en el grupo que interesa a este estudio, la presencia de aquellas experiencias que, ocurridas durante la infancia y la pubertad, diera, cuenta de ciertas fallas de importancia en la función del contexto cuidador de facilitar la integración de las experiencias afectivas de estos jóvenes. 
De los hallazgos encontrados podemos sostener que en este grupo de adolescentes obesos se observa en primer lugar la presencia evidente de sintomatología psíquica (ansiedad y depresión), en segundo lugar la presencia de afectos intensos (agresión, sentimientos de exclusión) pero a la vez una dificultad en la tolerancia a sus afectos (uso de mecanismos defensivos tendientes a disociar dichos afectos). Lo anterior conduce a que dichos afectos no son usados como señales de estados internos del sí mismo o del self (dada su condición de disociación) y por lo tanto siendo de muy poca utilidad para las experiencias personales de desarrollo y maduración.

Esto nos lleva a plantear que la obesidad (y el trastorno de la alimentación que subyace a este efecto) es una forma de expresión de una falla del contexto regulador que rodea al joven. Los adolescentes obesos tienen la percepción subjetiva de haber vivido experiencias afectivas intensas (primordialmente de pérdida) frente a las cuales no encontraron contención emocional. Postulamos que precede a la instalación de la obesidad en el cuerpo, una acumulación de experiencias de no sintonización afectiva del contexto parental. Las emociones y sentimientos encuentran en el trastorno corporal una forma de lograr un estado interno de equilibrio emocional.

Un aspecto interesante a discutir es el hallazgo positivo de sintomatología angustiosa y depresiva, la que contrade- ciría la habitual descripción alexitímica de los trastornos psicosomáticos.

\section{Referencias}

Alexander, F. (1952). La medicina Psicosomática. Paris : Payot .

Davis, M. \& Wallbridge, D. (1981). Limites y Espacio. Introducción a la obra de D. W. Winnicott. Buenos Aires: Editorial Amorrortu.

Krystal, H. (1988). lntegration and self-healing: affect, trauma, alexthymia. Hillsdale, N. J. The analytic Press.

Marty, P. (1992). La Psicosomática del Adulto. Buenos Aires: Editorial Paidós.

McDougall, J. (1991). Teatros del Cuerpo. España: Tecnipublicaciones.

McDougall, J. (1998). Las mil y una caras de Eros. Buenos Aires: Paidós.

McDougall, J. (1996). Alegato por una cierta anormalidad. Buenos Aires: Editorial Paidós.

Mitchell, S. (1993). Conceptos relacionales en psicoanálisis, una integración. Siglo XXI Editores.

Stolorow, R. Atwood, G. (1992). Contexts of being. The intersubejctive foundations and psychological life. New York: Analytic Press.

Taylor, G. (1987). Psychosomatic Medicine and Contemporary Psychoanalysis. Conneticut: International University Press.

Winnicott, D. W. (1949). Escritos de pediatría y psicoanálisis. Barcelona: Ediciones Paidós.

Winnicott, D. W. ( 1954). La naturaleza Humana. Buenos Aires: Editorial Paidós.

Winnicott, D. W. (1964). Exploraciones Psicoanalíticas. Buenos Aires: Editorial Paidós. 